\title{
ON SEPARABLE EXTENSIONS OF GROUP RINGS AND QUATERNION RINGS
}

\author{
GEORGE SZETO \\ Mathematics Department \\ Bradley University \\ Peoria, Illinois 61625 U.S.A.
}

(Received November 29, 1977)

ABSTRACT. The purposes of the present paper are (1) to give a necessary and sufficient condition for the uniqueness of the separable idempotent for a separable group ring extansion RG ( $R$ may be a non-commutative ring), and (2) to give a full description of the set of separable idempotents for a quaternion ring extension $R Q$ over a ring $R$, where $Q$ are the usual quaternions $i, j, k$ and multiplication and addition are defined as quaternion algebras over a field. We shall show that RG has a unique separable idempotent if and only if $G$ is abelian, that there are more than one separable idempotents for a separable quaternion ring $R Q$, and that $R Q$ is separable if and only if 2 is invertible in R.

KEY WORDS AND PHRASES. Group Rings, Idempotents in Rings, Separable Algebras AMS (MOS) SUBJECT CLASSIFICATION (1970) CODES. Major: 16A26 Minor: 16A16, $16 \mathrm{~A} 32$ 


\section{INTRODUCTION.}

M. Auslander and 0 . Goldman ([1] and [2]) studied separable algebras over a commutative ring. Subsequently, the investigation of separable algebras (in particular, Brauer groups and Azumaya algebras) has attracted a lot of researchers, and rich results have been obtained (see References). K. Hirata and K. Sugano ([5]) generalized the concept of separable algebras to separable ring extensions; that is, let $S$ be a subring of a ring $T$ with the same identity. Then $T$ is called a separable ring extension of $S$ if there exists an element $\sum a_{i} \otimes b_{i}$ in $T \otimes_{S} T$ such that $x\left(\sum a_{i} \otimes b_{i}\right)=\left(\sum a_{i} \otimes b_{i}\right) x$ for each $x$ in $T$ and $\sum a_{i} b_{i}=1$. Such an element $\Sigma a_{i} \otimes b_{i}$ is called a separable idempotent for $T$. We note that a separable idempotent takes an important role in many theorems (for example, see [6], Section 5,6, and 7). It is easy to verify that $(1 / n)\left(\sum g_{i} \otimes g_{i}^{-1}\right)$ and $\sum e_{i 1} \otimes e_{1 i}([4]$, Examples II and III, P. 41) are separable idempotents for a group algebra $R G$ and a matrix $\operatorname{ring} M_{m}(R)$ respectively, where $G=\left\{g_{1}, \ldots, g_{n}\right\}$ with $n$ invertible in $R$ and $e_{i j}$ are matrix units. We also note that the separable idempotent for a commutative separable algebra is unique ([6], Section 1, P. 722).

\section{PRELIMINARIES.}

Throughout, $G$ is a group of order $n, R$ is a ring with an identity 1. The group ring $R G=\left\{\sum r_{i} g_{i} / r_{i}\right.$ in $R$ and $g_{i}$ in $\left.G\right\}$, which is a free R-module with a basis $\left\{g_{i}\right\}$ and $\left(\sum r_{i} g_{i}\right)\left(\sum s_{i} g_{i}\right)=\sum t_{k} g_{k}$ where $t_{k}=$ $\sum r_{i}{ }_{j}$ for all possible $i, j$ such that $g_{i} g_{j}=g_{k}$. The ring $R$ is imbedded in $R G$ by $r \rightarrow r g_{1}$, where $g_{1}$ is the identity of $G\left(g_{1}=1\right)$. The multiplication map $R G \otimes_{R} R G \rightarrow R G$ is denoted by $\pi$. Clearly, $\left\{g_{i} \otimes_{j} / i, j=\right.$ 
$1, \ldots, n\}$ form a basis for $R G \otimes_{R} R G$. An element $\sum r_{i j}\left(g_{i} \otimes_{j}\right)$ in $R G \otimes_{R} R G$ is called a commutant element in $R G \otimes_{R} R G$ if $x\left(\sum r_{i j}\left(g_{i} \otimes_{j}\right)\right)=$ $\left(\sum r_{i j}\left(g_{i} \otimes g_{j}\right)\right) x$ for all $x$ in $R G$.

\section{MAIN THEOREMS.}

We begin with a representation for $\pi(x)$ for a commutant element $x$ in $R G \otimes_{R} R G$, and then we show that $R G$ has a unique separable idempotent if and only if $G$ is abelian.

LEMMA 1. Let $x=\sum r_{i j}\left(g_{i} \otimes g_{j}\right), i, j=1, \ldots, n$, be a commutant element in $R G \otimes_{R} R G$. Then $\pi(x)=\sum_{i=1}^{m}\left(\sum r_{1 k_{i}}\right) n_{k_{i}} c_{k_{i}}$, where $m$ is the number of conjugate classes of $\mathrm{G}, \mathrm{n}_{\mathrm{k}_{i}}$ is the order of the normalizer of $E_{k_{i}}$, and $\mathrm{C}_{\mathrm{k}_{i}}$ is the sum of different conjugate elements of $\varepsilon_{k_{i}}$, for some $k_{i}$ and $k_{i}$ in $\{1, \ldots, n\}$

PROOF. Since $x$ is a commutant element, $g_{p} x=x g_{p}$ for each $g_{p}$ in $G$. The coefficient of the term $g_{p} g_{k}$ in $g_{p} x$ is $r_{1 k}$, and the coefficient of the same term in $x g_{p}$ is $r_{p q}$, where $g_{q} g_{p}=g_{k}$. Hence $r_{1 k}=r_{p q}$ whenever $g_{q} g_{p}=g_{k}$. Thus $x=\sum_{k} r_{1 k}\left(\sum g_{p} g_{q}\right)$, where $p, q$ run over $1, \ldots, n$, such that $g_{q} g_{p}=g_{k} ;$ that is, $x=\sum_{k} r_{1 k}\left(\sum_{p} g_{p} g_{k} g_{p}^{-1}\right)$. Taking $\pi(x)=$ $\Sigma_{k} r_{1 k}\left(\sum_{p} g_{p} g_{k} g_{p}^{-1}\right)$. For a fixed $k, \sum_{p}{ }^{E} g_{k} g_{p}^{-1}=n_{k} c_{k}$ where $n_{k}$ is the order of the normalizer of $g_{k}$ and $C_{k}$ is the sum of all different conjugate elements of $g_{k}$. Hence $\pi(x)=\sum_{k=1}{ }_{n}{ }_{1 k} n_{k} C_{k}$. Since conjugate classes form a partition of $G, C_{i}=C_{j}$ if and only if $g_{i}$ is conjugate to $E_{j}$. Renumerating elements, we let $\left\{g_{k_{1}}, \ldots, g_{k_{m}}\right\}$ be all non-conjugate elements of each other; then $\left\{\mathrm{C}_{\mathrm{k}_{1}}, \ldots, \mathrm{C}_{\mathrm{k}_{\mathrm{m}}}\right\}$ are all different elements in the set, $\left\{c_{1}, \ldots, c_{n}\right\}$. Thus $\pi(x)=\sum_{i=1}^{m}\left(\sum r_{1 k_{i}}\right) n_{k_{i}} c_{k}$, where $r_{1 k}$, are coefficients of the same $\mathrm{C}_{\mathrm{k}_{i}}$, and $\mathrm{m}$ is the number of conjugate classes 
of $G$.

THEOREM 2. Let $R G$ be a separable extension of $R$. Then, RG has a unique separable idempotent if and only if $G$ is abelian.

PROOF. Let $\mathrm{x}=\boldsymbol{\Sigma} \mathrm{r}_{i j}\left(\mathrm{~g}_{i} \otimes g_{j}\right)$ be a separable idempotent for RG. Then by the lemma, $\pi(x)=\sum_{i=1}^{m}\left(\sum r_{1 k_{i}^{\prime}}\right) n_{k_{i}} c_{k_{i}}$, where $c_{k_{i}}$ is the sum of all conjugate elements of $g_{k_{i}}$. Let $g_{k_{1}}=1$, the identity of $G$. Then

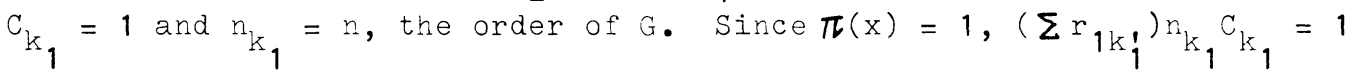
and $\left(\sum r_{1 k_{1}}\right) n_{k_{1}}{ }^{c_{k}}=1$ and $\left(\sum r_{1 k_{i}}\right) n_{k_{i}} C_{k_{i}}=0$ for each $i \neq 1$. Noting that $c_{k_{1}}=1$, we have $\sum r_{1 k_{1}}=r_{11}$, and so the first equation becomes $r_{11} n=1$. Hence the order of $G, n$, is invertible in $R$. Thus $n_{k_{i}}$, being a factor of $\mathrm{n}$, is also invertible in $\mathrm{R}$. But conjugate classes form a partition of $G$, so $\left(\sum r_{1 k_{i}}\right) n_{k_{i}} C_{k_{i}}=0$ implies that $\sum r_{1 k_{i}}=0$ for each $i \neq 1$. This system of homogeneous equations $\sum r_{1 k_{i}}=0$ in the unknowns $r_{1 k}$ with $i \neq 1$ has trivial solutions if and only if $n=m$, and this holds if and only if $G$ is abelian. Since the uniqueness of the separable idempotent $\left(=(1 / n)\left(\sum g_{i} \otimes g_{i}^{-1}\right)\right)$ is equivalent to the existence of trivial solutions of the above system of equations, the same fact is equivalent to $G$ being abelian.

The theorem tells us that there are many separable idempotents for a separable group ring $R G$ when $G$ is non-abelian. Also, we remark that if $R G$ is a separable extension of $R$, the order of $G$ is invertible in $R$ from the proof of the theorem. Next, we discuss another popular separable ring extension, a quaternion ring extension $R Q$, where $R Q=$ $\left\{r_{1}+r_{i} i+r_{j} j+r_{k} k / i, j\right.$, and $k$ are usual quaternions $\} \cdot\left(R Q_{2}+\cdot\right)$ is a ring extension of $R$ under the usual addition and multiplication similar to quaternion algebras over a field. Now we characterize a separable idem- 
potent for a separable quaternion ring extension $R Q$.

THEOREM 3. Let $R Q$ be a separable quaternion ring extension. Then a commutant element $x=\sum r_{s t}(s \otimes t), s, t=1, i, j, k$, in $R Q \otimes_{R} R Q$ is a separable idempotent for $R Q$ if and only if $r_{11}=1 / 4$.

PROOF. Since $x$ is a commutant element in $R Q Q_{R} R Q$, $i x=x i$. The coefficients of the term $1 \otimes 1$ on both sides are $-r_{i 1}$ and $-r_{1 i}$, so $r_{i 1}=r_{1 i}$. Since $j x=x j$, the coefficients of the term $k \otimes 1$ on both sides are $-r_{i 1}=$ $-r_{k j}$, so $r_{i 1}=r_{k j}$. Also, $k x=x k$, so the coefficients of the term $j \otimes 1$ on both sides are $-r_{i 1}=r_{j k}$. Hence $r_{1 i}=r_{i 1}=r_{k j}=-r_{j k}$. Similarly, by comparing coefficients of other terms, we have $r_{11}=-r_{i i}=-r_{j j}=$ $-r_{k k}, r_{1 j}=r_{j 1}=-r_{k i}=r_{i k}$ and $r_{1 k}=r_{k 1}=-r_{i j}=r_{j i}$. In other words, $r_{s t}=r_{p q}$ if $t s=q p$, and $r_{s t}=-r_{p q}$ if $t s=-q p$. Thus

$x=r_{11}(1 \otimes 1-i \otimes i-j \otimes j-k \otimes k)+r(i \otimes i+i \otimes 1-j \otimes k+k \otimes j)+r_{1 j}(1 \otimes j+j \otimes 1-k \otimes i+i \otimes k)+$

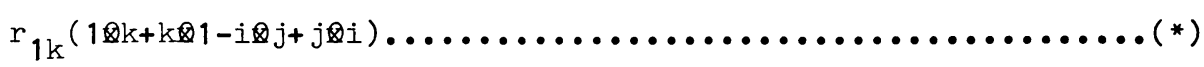

But then $\pi(x)=r_{11^{4+r}}{ }_{1 i}{ }^{0+r_{1 j}}{ }^{0+r_{1 k}}{ }^{0}=4 r_{11}$. Consequently, $x$ is a separable idempotent if and only if $r_{11}=1 / 4($ for $\pi(x)=1)$.

COROLLARY 4. Let $R Q$ be a quaternion ring extension of $R$. Then $R Q$ is separable if and only if 2 is invertible in $R$.

PROOF. The necessity is immediate from the theorem. The sufficiency is clear since the element $x$ with $r_{11}=1 / 4, r_{1 i}=r_{1 j}=r_{1 k}=0$ as given in (*) in Theorem 3 is a separable idempotent for $R Q$.

REMARK. It is easy to see that every $x$ of the form (*) in Theorem 3 with $r_{11}, r_{1 i}, r_{1 j}$ and $r_{1 k}$ in the center of $R$ is a commutant element in $R Q Q_{R} R Q$. Hence, from the proof of Theorem 3, the complete set of commutant elements is: $\quad C=\left\{\Sigma_{s t}(s \otimes t) / r_{s t}=r_{p q}\right.$ if $q p=t s$, and $r_{s t}=$ $-r_{p q}$ if $\left.q p=-t s\right\}$. Also, the complete set of separable idempotents for 
$R Q$ is a subset of $C$ such that $r_{11}=1 / 4$ and $r_{1 i}, r_{1 j}, r_{1 k}$ are in the center of $R$. Thus there are many separable idempotents.

\section{REFERENCES}

1. Auslander, M. and O. Goldman. The Brauer Group of a Commutative Ring, Trans. Amer. Math. Soc. 97 (1960) 367-409.

2. Auslander, M. and O. Goldman. Maximal Orders, Trans. Amer. Math. Soc. 27 (1960) 1-24.

3. Bass, H. Lectures on Topics in Algebraic K-Theory, Tata Institute of. Fundamental Research, Bombay, 1967.

4. DeMeyer, F. and E. Ingraham. Separable Algebras Over Commutative Rings, Springer-Verlag, Berlin-Heidelberg-New York, 181, 1971.

5. Hirata, K. and K. Sugano. On Semisimple Extensions and Separable Extensions over Non-Commutative Rings, J. Math. Soc. Japan 18 (1966) 360-373.

6. Villamayor, 0 . and D. Zelinsky. Galois Theory for Rings with Finitely Many Idempotents, Nagoya Math. J. 27 (1966) 721-731.

7. Zelinsky, D. Brauer Groups, Springer-Verlag, Berlin-HeidelbergNew York, 549, 1976. 


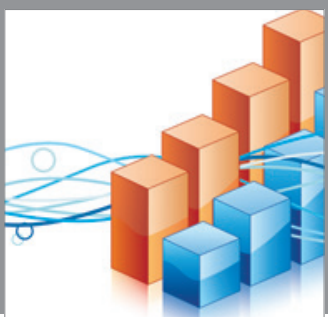

Advances in

Operations Research

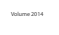

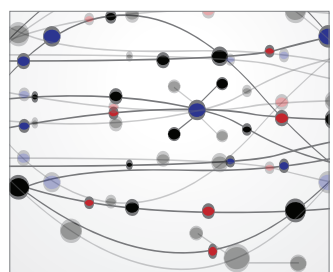

\section{The Scientific} World Journal
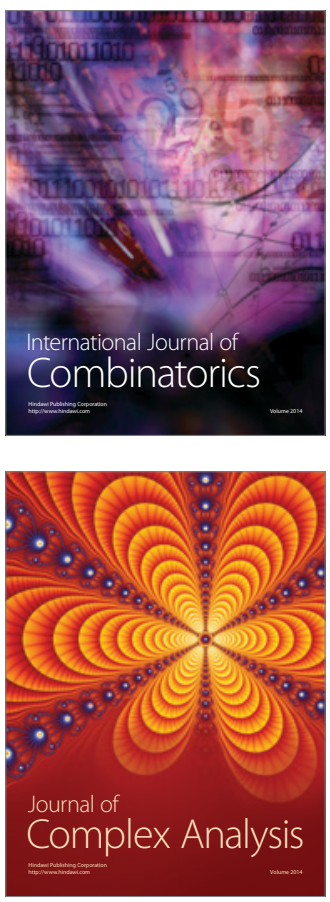

International Journal of

Mathematics and

Mathematical

Sciences
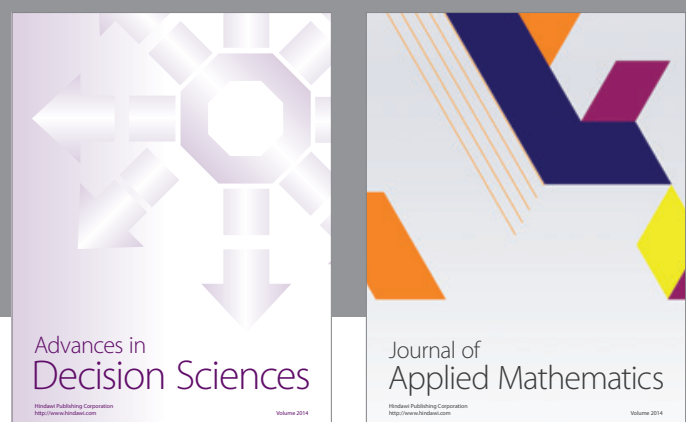

Journal of

Applied Mathematics
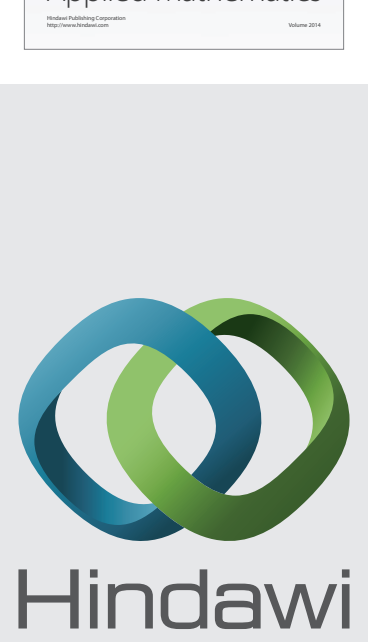

Submit your manuscripts at http://www.hindawi.com
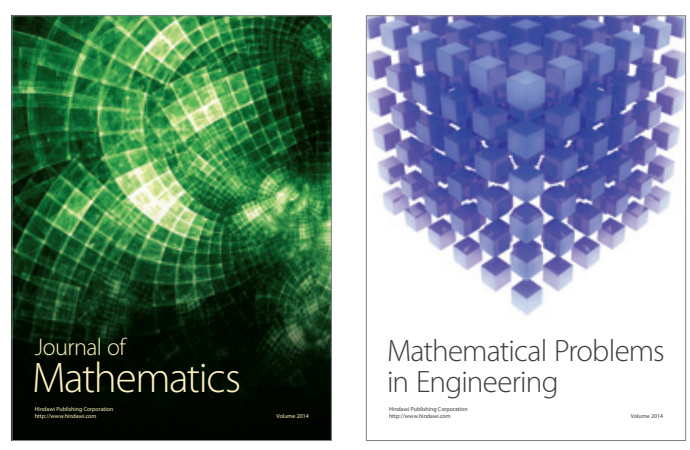

Mathematical Problems in Engineering
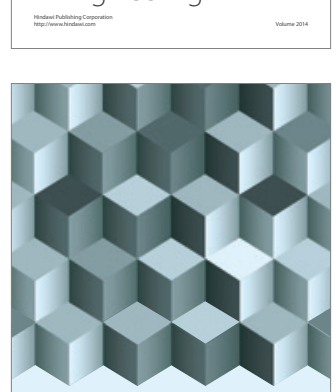

Journal of

Function Spaces
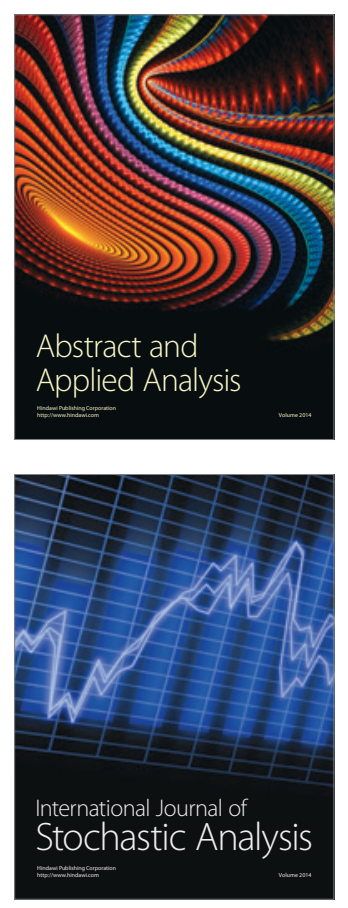

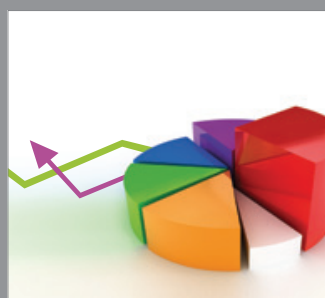

ournal of

Probability and Statistics

Promensencen
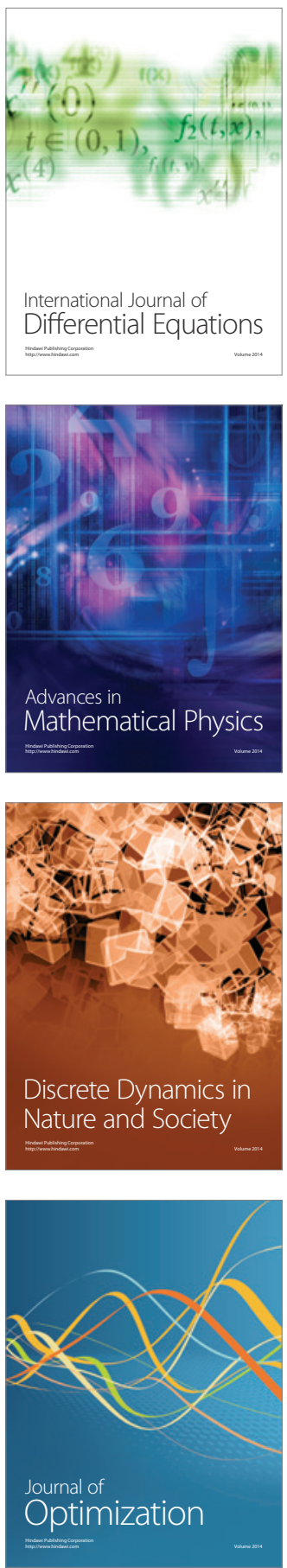